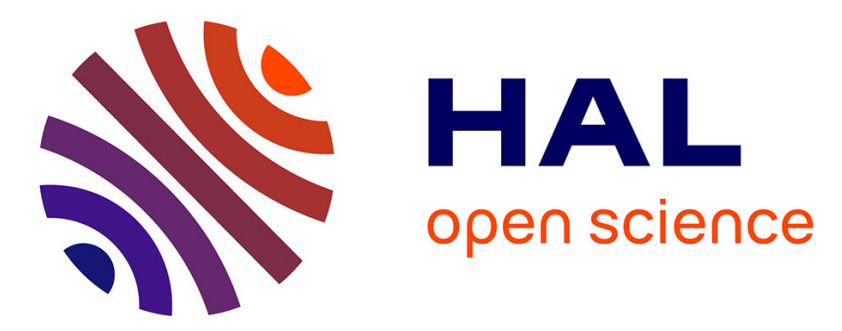

\title{
Ultrasound Molecular Imaging with Targeted Microbubbles for Cancer Diagnostics: From Bench to Bedside
}

\author{
J. Wischhusen, F. Padilla
}

\section{- To cite this version:}

J. Wischhusen, F. Padilla. Ultrasound Molecular Imaging with Targeted Microbubbles for Cancer Diagnostics: From Bench to Bedside. Innovation and Research in BioMedical engineering, 2019, 40, pp.3 - 9. 10.1016/j.irbm.2018.10.007 . hal-03484596

\author{
HAL Id: hal-03484596 \\ https://hal.science/hal-03484596
}

Submitted on 20 Dec 2021

HAL is a multi-disciplinary open access archive for the deposit and dissemination of scientific research documents, whether they are published or not. The documents may come from teaching and research institutions in France or abroad, or from public or private research centers.
L'archive ouverte pluridisciplinaire HAL, est destinée au dépôt et à la diffusion de documents scientifiques de niveau recherche, publiés ou non, émanant des établissements d'enseignement et de recherche français ou étrangers, des laboratoires publics ou privés.

\section{(ㄷ)(1) $\$$}

Distributed under a Creative Commons Attribution - NonCommercial| 4.0 International 


\section{Authors:}

4 Jennifer Wischhusen ${ }^{1,2}$, Frederic Padilla ${ }^{1,3,4}$

5 Affiliations:

6 1: Univ Lyon, Université Lyon 1, Centre Léon Bérard, INSERM, LabTAU, F-69003, LYON,

7 France

8 2: Apoptosis, Cancer and Development Laboratory - Equipe labellisée 'La Ligue', LabEx

9 DEVweCAN, Centre de Cancérologie de Lyon, INSERM U1052-CNRS UMR5286, Centre Léon

10 Bérard, 69008 Lyon, France

11 3: Focused Ultrasound Foundation, Charlottesville, USA

12 4: Radiation Oncology, University of Virginia School of Medicine, USA

14 Address:

15 LabTAU INSERM U1032, 151 cours Albert Thomas, 69003 Lyon

16 Mobile: +33778210650

17 E-mail: Frederic.Padilla@inserm.fr

18 E-Mail : Jennifer.Wischhusen@1yon.unicancer.fr 


\section{Abstract}

21 Background: Ultrasound plays an important role in cancer diagnosis. B-mode imaging and contrast22 enhanced ultrasound are routinely used to detect cancerous lesions in breast and liver. The use of 23 ultrasound contrast agents (UCAs) such as microbubbles (MBs), which can be functionalized with 24 targeting ligands, has further enabled ultrasound molecular imaging (USMI) of specific molecular 25 markers in pre-clinical and the first clinical studies. As targeted MBs have a diameter of 1-4 $\mu \mathrm{m}$, they 26 are limited to the blood vasculature upon intravenous injection, and can bind to markers of the 27 vascular endothelium. USMI with targeted MBs was applied for imaging of markers of inflammation, 28 angiogenesis, and the tumor endothelium.

29 Aim: The present review provides an introduction to USMI and presents currently available UCAs, 30 targeting strategies, pre-clinical targets, proposed applications, and the first clinical studies with 31 USMI to guide novel users and assess the technique's potential for clinical use.

32 Key points:

- USMI provides information about markers that are useful for cancer diagnostics.

34 - UCAs are functionalized with targeting ligands for molecular imaging.

35 - Imaging strategies allow distinction between circulating and attached MBs.

36 - Targeted MBs are used for cancer angiogenesis imaging in pre-clinical studies.

- BR55 proved the feasibility of clinical cancer imaging by USMI.

38

39 Graphical abstract: 


\section{Targeted microbubble}

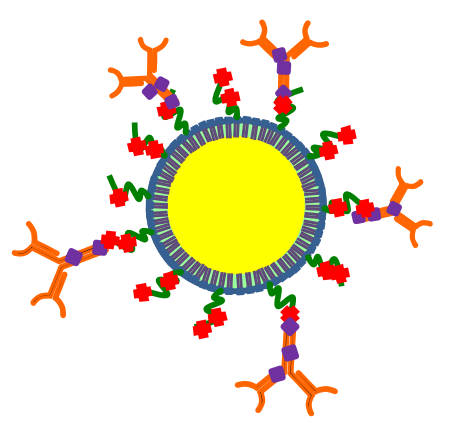

40

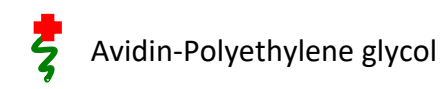

Biotinylated antibody
Ultrasound molecular imaging

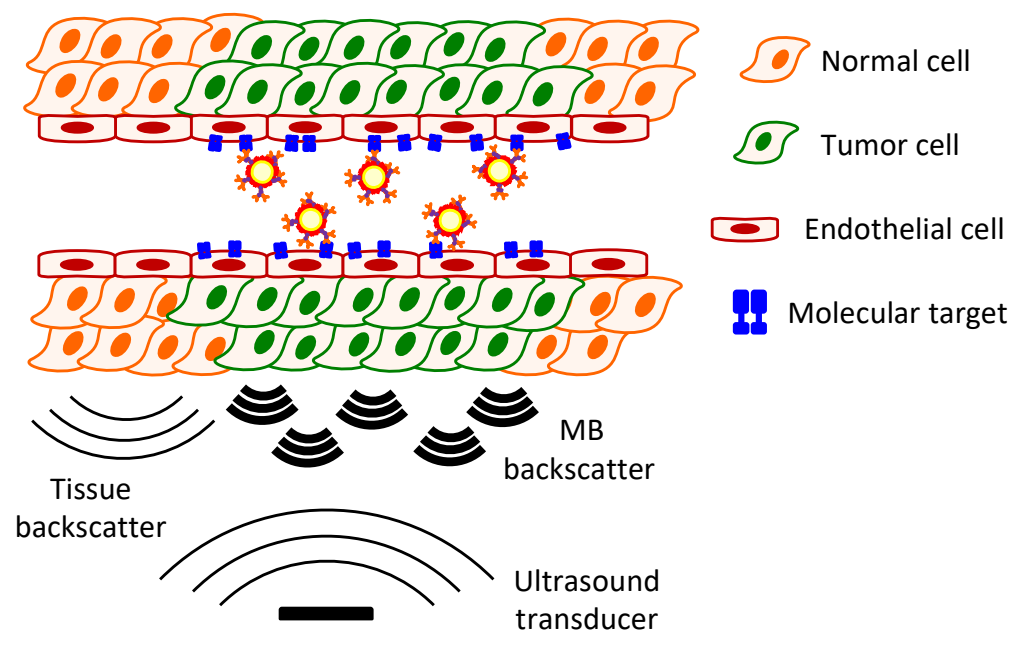




\section{Ultrasound imaging}

Ultrasound is widely used for biomedical imaging. It is safe, cost-effective, non-invasive,

44 can be used at the bed side, provides images in real-time, and does not produce ionizing radiation.

45 Limitations in the use of ultrasound for diagnostic purposes are tissues containing gases or bones

46 as they interfere with ultrasound propagation. Ultrasound allows imaging up to $15 \mathrm{~cm}$ deep into

47 the body at the classical frequency range of 1-20 $\mathrm{MHz}$ [1]. Depending on the ultrasound transducer and the generated pulse, spatial resolution varies from 0.1 to $1.5 \mathrm{~mm}$ [1]. Ultrasound imaging is based on the principle that longitudinal sound waves are sent out into the tissue and get reflected by structures with differences in impedance. This creates an echo that can be detected and resolved to depict tissue anatomy in so-called brightness mode (B-mode) [1]. develop into abnormal masses of tissue. Elastography, an ultrasound imaging approach that detects tissue stiffness based on the propagation of shear waves [1], can also be used for cancer diagnostic as tumor tissue stiffness has been shown to differ from surrounding normal tissue.

Besides anatomical imaging, ultrasound can provide functional imaging, such as blood flow, based on Doppler, a shift in frequency of the backscattered longitudinal acoustic waves due to motion [2] that can be used to extract information about blood flow. Blood flow information is particularly interesting in tumors as tumoral blood vessels differ from normal blood vessels because tumor angiogenesis induces a partly immature, disorganized vasculature in which the blood flow is disturbed [3].

In case of slowed blood flow, contrast-enhanced ultrasound (CEUS) can be applied to improve cancer detection and tumor characterization [4]. CEUS requires the use of ultrasound contrast agents (UCAs). The first FDA-approved UCA was an albumin-coated and air-filled microsphere [5]. Since then, UCAs have been largely improved regarding stability and safety. 
Today, UCAs are mostly microbubbles (MBs), which have a diameter in the micrometer range and consist of a gas core most often stabilized by a lipid shell containing polyethylene glycol (PEG) molecules for stearic protection (Fig. 1) [6]. Due to their size, MBs are limited to the blood circulation upon intravenous injection. Thus, MBs are intravascular contrast agents which are used to assess functional perfusion parameters such as vessel sizes, density, and flow rate [4]. To distinguish MBs from tissue, specific ultrasound imaging sequences were developed exploiting the fact that tissue shows a linear response to ultrasound while MBs have a non-linear response [7].

MBs were developed further and conjugated with targeted ligands to enable ultrasound molecular imaging (USMI) [8]. The ligand can be coupled to the MB shell by different methods such as non-covalent avidin-biotin bridge if MBs carry avidin molecules on PEG and/or lipids and get incubated with biotinylated antibodies [9]. Recently, a clinical-grade targeted UCA was developed, named BR55, which is targeted towards the human kinase insert domain receptor (KDR) or CD309 and cross-reacts with murine Vascular Endothelial Growth Factor Receptor (VEGFR2) owing to a peptide ligand that is covalently attached to the lipid shell [10]. Targeted MBs have the capacity to recognize and bind molecular markers that are expressed on the vascular endothelium (Fig. 2). USMI was therefore proposed for diagnosis and monitoring of pathological processes involving the endothelium [9].

In order to discriminate free-circulating and attached MBs from each other, different imaging and data treatment approaches have been developed such as the destructionreplenishment method where MBs are destroyed to detect residual free-circulating MBs and calculate the targeted MB signal by signal subtraction, or late phase imaging where images are acquired during the MB wash-out phase so that only specifically bound MBs are left in the image [11]. 
Recent advances in computer technology and software enabled the implementation of

91 three-dimensional real-time ultrasound imaging in the clinic [12]. Three-dimensional ultrasound

92

93

94 provides a more complete anatomic assessment for better reliability in diagnosis and disease monitoring. This imaging approach was also combined with CEUS and USMI and proved useful for early and precise therapy response assessment upon anti-tumor therapy [13].

Ultrasound and CEUS are integral components of clinical disease management for different cancers, including breast and liver, which distinguish cancer from normal tissues as abnormal masses with uncoordinated vasculature. As the causes of cancer are aberrantly expressed proteins that dysregulate cellular proliferation and survival, molecularly targeted therapies were developed to specifically attack the tumor cells while preserving normal tissue. Information provided by B-mode or CEUS imaging about the location, size, and perfusion of the tumor are not sufficient to predict the patient's response to a targeted therapy. USMI has the potential to visualize the expression level and distribution of tumor-related vascular markers and allow for treatment decision and early monitoring of the therapeutic response.

\section{Ultrasound contrast agents}

\section{Microbubbles}

Microbubbles (MBs) are the most commonly used UCAs. Their size ranges from 1-4 $\mu \mathrm{m}$ in diameter and they are composed of a gas core stabilized by a shell made of phospholipids, polymers or proteins [14]. The echogenic gas can be air, nitrogen, or inert gases such as perfluorocarbons, which increase the half-life of MBs [15]. Protein- and lipid-shelled MBs are prepared through gas dispersion into protein or lipid suspension under high-shear mixing [14]. In the case of polymeric MBs, an organic phase containing the polymer is emulsified in an aqueous phase, and the solvent is evaporated [16]. Usually polyethylene glycol (PEG) is added to the shell 
114 formulation to increase stability and reduce uptake by the reticuloendothelial system (RES) [4].

115 Several types of MBs are currently approved for clinical use including lipid-shelled 116 Definity/Luminity, Lumason/SonoVue, Sonazoid, and albumin-shelled Optison [4]. There are 117 used for diagnosis of breast, kidney, and liver lesions [17]. MBs are provided as lyophilized 118 powder, which has to be re-suspended prior to use. Upon injection, MBs have a relatively short 119 half-life ranging from a few minutes up to $30 \mathrm{~min}$ for the different MB types [17]. MBs are 120 retained in lung, liver and spleen and they degrade in small vessels, although a partial 121 redistribution after lung passage was reported [17]. The gas of disintegrated MBs is exhaled 122 through the lungs, shell components are eliminated by kidneys or phagocytosed by macrophages $123[17]$.

\section{Alternative contrast agents}

Alternatively to the described intravascular UCAs, nanometer-sized UCAs were more recently proposed, that could passively extravasate into the tumor tissue through the enhanced 127 permeability and retention (EPR) effect (Fig. 3) [17]. Echogenic liposomes composed of a 128 phospholipid bilayer in which air or other gases are entrapped were developed [18]. As these 129 liposomes can be loaded with drugs, a theranostic application of these agents is possible [17]. 130 Other forms of nanometer-sized UCAs are nanobubbles, nanodroplets and solid nanoparticles. 131 Nanobubbles are composed of gas, liquid, or solid cores stabilized by lipid or polymer shells and 132 prepared in a more complex protocol compared with $\mathrm{MBs}$, to avoid coalescence and preserve 133 small sizes [17]. Further, nanobubbles synthesis is associated with low purity, instability and 134 increased toxicity, which renders the use of these agents less attractive for clinical applications 135 [17]. Initially, size reduction rendered the first generation of these novel UCAs unstable and less 136 echogenic than MBs [19]. Today, novel formulations of echogenic liposomes and nanobubbles 137 have been reported with similar echogenicity than MBs [20]. A last class of nanoscale UCAs are 
138 phase-change contrast agents (PCCAs) which are droplets with a liquid perfluorocarbon core 139 stabilized by lipid, protein or polymer shells [21]. Perfluorocarbons are used that have boiling 140 points close to or below body temperature. They are kept in liquid state even after injection due 141 to high pressure in the small stabilized droplet. Upon ultrasound exposure, perfluorocarbons 142 switch into gas state, which results in a 4-10 times expansion in size, and strong echogenic 143 response. PCCA can be prepared by different techniques such as MB condensation, microfluidics 144 or emulsification. The conversion of nanodroplets into MBs causes cavitation and sonoporation, 145 suggesting a potential role of PCCAs for drug delivery applications [17].

\section{Molecular targeting approaches}

Non-covalent and covalent targeting strategies are now described. Non-covalent

149 functionalization of $\mathrm{MBs}$ includes the incorporation of targeting ligands, such as 150 phosphatidylserine, which mediates binding to activated leukocytes, and proteins, into the UCA 151 shell [17]. Further, the use of linker molecules such as biotinylated lipids or avidinylated PEG 152 arms is very common to provide an interface for functionalization [22]. The use of non-covalent 153 biotin-avidin functionalization strategies is limited by the risk of immunogenicity thus preventing 154 the use of these UCAs in clinic. Covalent targeting methods are based on the reactions with the 155 chemical groups exposed on the MB surface. Carboxyl groups of UCAs can react with amine groups of proteins and form amide bonds, and maleimide-containing lipids and thiol-activated 157 ligands can result in thiol-maleimide bonds [17]. Targeting ligands include antibodies, peptides, 158 natural and engineered scaffolds [14]. The first clinical-grade targeted UCA BR55 was 159 formulated using a heterodimer peptide with specificity for VEGFR2 that was covalently 160 attached to a lipid-PEG construct through its amino group [10]. 


\section{Pre-clinical targets and applications}

The most studied target for USMI with MBs has been VEGFR2 until now. VEGFR2 is

164 the receptor of Vascular Endothelial Growth Factor (VEGF) and the main regulator of vascular 165 endothelial cell development under physiological conditions, and tumor angiogenesis in pathological conditions [23]. The inhibition of VEGFR2 and tumor angiogenesis was proposed as

167 a therapeutic strategy, and several drugs were developed that interfere with VEGFR2 signaling

168 [23]. Pre-clinical-grade anti-VEGFR2-MBs were used in studies detecting angiogenesis, and 169 evaluating tumor angiogenesis over time [24]. Clinical-grade anti-VEGFR2-MBs were studied 170 for cancer detection, assessment of tumor angiogenesis and aggressiveness, therapy monitoring, 171 and discrimination of different anti-angiogenic therapies [10,25]. transmembrane glycoprotein involved in embryonic but also in tumor angiogenesis [26], endoglin

174 (CD105), an accessory receptor for transforming growth factor receptor beta mediating 175 endothelial cell proliferation, angiogenesis and neovascularization [27], and nucleolin, which is 176 correlated with tumor aggressiveness [28].

177 E-selectin, P-selectin, intercellular adhesion molecule-1 (ICAM-1), vascular cellular 178 adhesion molecule-1 (VCAM-1), junctional adhesion molecule-A (JAM-A), and integrins were 179 also repeatedly studied as targets for USMI. These adhesion molecules are cell surface 180 glycoproteins mediating intercellular contact, and contact between cells and the extracellular 181 matrix. They regulate immune cell responses and cell migration in inflamed tissues [29]. 182 Moreover, these proteins are involved in pathological conditions involving inflammation and 183 immune cells such as ischemia-reperfusion injury, transplant rejection, inflammatory diseases of 184 the cardiovascular system, skin, kidneys, gastrointestinal tract, brain and liver [29]. P-selectin185 targeted MBs were used for inflammation imaging [30]. For inflammation imaging and anti- 
angiogenic therapy monitoring, anti-E-selectin MBs were used [31]. ICAM-1-targeted MBs indicated vascular responses in tumor therapy, and VCAM-1-targeted MBs revealed vessel healing after surgery [32]. Anti-JAM-A-MBs were used to image vulnerable plaque [33]. Integrin $\alpha_{v} \beta_{3}$ was imaged to detect vessel injury, therapy response, angiogenesis, and early atherosclerotic plaque [34]. Integrin $\alpha_{\mathrm{IIb}} \beta_{3}$ (glycoprotein IIb/IIIa receptor) was used as a marker of angiogenesis [35].

Different cardiovascular targets were also selected for USMI including the glycoprotein IV (CD36), which is a collagen receptor involved in platelet aggregation and adhesion [36], thrombin which regulates homeostasis through pro- and anti-coagulant activities [37], and CD81 and glycoprotein Ib $\alpha$ which are upregulated in atherosclerotic plaque and can allow their early detection [38].

Several tumor-type-specific targeted MBs were also developed and evaluated in preclinical USMI studies. CD276 (B7-H3) is a transmembrane protein, an immune checkpoint protein ubiquitously expressed in non-lymphoid and lymphoid organs and overexpressed in human cancers, was used for breast cancer detection by USMI [39]. IL-16, an inflammatory cytokine, was studied as a marker of early ovarian cancer [40]. Another tumor-specific imaging approach was developed using pimonidazole to label hypoxic tumors, and pimonidazole-targeted MBs for detection [41]. Secreted frizzled related protein 2 (SFRP2) was imaged with MBs to detect angiosarcoma [42]. Prostate specific membrane antigen (PSMA)-targeted MBs were developed for prostate cancer detection [43]. Netrin-1, a secreted ligand binding to dependence receptors and triggering survival and proliferation, was imaged with MBs in breast tumors (Figure 4) [44]. 
Dual- and triple-targeted MBs were also proposed combining targeting to VEGFR2 and $\alpha_{v} \beta_{3}$ integrin [45], P-selectin and E-selectin [46], P-selectin and VCAM-1 [47], and to VEGFR2, P-selectin and $\alpha_{\mathrm{v}} \beta_{3}$ integrin [48].

\section{Imaging strategies}

The introduction of UCAs required the development of imaging strategies that could distinguish the signals backscattered by MBs from the signal backscattered by the tissue. MBs oscillate nonlinearly, i.e. that the amplitude and phase of the reflected ultrasound pulses are distorted compared to tissue echoes [11]. Multi-pulse techniques for UCA detection were developed relying on the separation of nonlinear MB responses from linear tissue responses. Pulse inversion, contrast pulse sequencing, amplitude modulation techniques, and combinations of both or derivations of them are most often used [11].

In USMI, bound MBs have to be discriminated from free-circulating MBs and tissue. Upon MB injection, a delay of several minutes is included to give time for the MBs to circulate and find their target. A high-intensity pulse is applied to destroy all MBs in the field of the ultrasound transducer. As MBs from other regions in the body replenish the imaged region, the signal of bound MBs is calculated by subtracting the post-burst images (depicting only the freecirculating MBs) from the pre-burst images (with free-circulating and bound MBs) [7]. Most of the reported preclinical USMI studies made use of the contrast pulse sequencing technique in combination with the destruction-replenishment method to specifically detect targeted MBs [7]. However, this technique requires a high-intensity pulse to destroy MBs so that a safety evaluation is required prior to clinical translation. Alternatively, signal from bound MBs can be determined by acquiring signal intensities at the peak of $\mathrm{MB}$ concentration in the target region and $7 \mathrm{~min}$ after injection during MB wash-out, and normalizing the late signal by the peak signal [7]. 
232 Recently, novel image processing techniques were proposed to specifically detect bound MBs,

233 which remain in the same location in different imaging frames while signal of free-circulating

234 MBs get suppressed. Another algorithm was developed to measure MB dwell time in near real235 time, and minimum intensity projection calculations were described [28].

\section{Clinical studies}

BR55 is the first FDA-approved targeted UCA with specificity for VEGFR2

239 (ClinicalTrials.gov identifier: NCT02142608) and was applied in a first safety and feasibility

240 pilot study for prostate cancer detection and VEGFR2 expression assessment in breast and

241 ovarian lesions $[49,50]$. Bound MBs were detected by measuring the late phase enhancement,

242 after free-circulating MBs have been supposedly cleared out. BR55 was shown to be safe for

243 USMI in patients with prostate, breast, or ovarian lesions. In prostate cancer patients, $68 \%$ of

244 malignant lesions were detected with BR55 [49]. In patients with ovarian and breast lesions, 77\%

245 of malignant ovarian cancer lesions and 93\% of malignant breast cancer lesions showed strong

246 imaging signal with BR55 while there was no molecular imaging signal in $78 \%$ of benign ovarian

247 lesions and in $67 \%$ of benign breast lesions [50]. Thus, molecular imaging with BR55 seems to

248 have high sensitivity and good specificity. USMI with BR55 might therefore allow clinical 249 translation for non-invasive detection and characterization of cancer.

\section{Conclusions}

Ultrasound molecular imaging (USMI) is a powerful technique for the detection of 253 endothelial markers involved in inflammation, cardiovascular disease, and tumor angiogenesis. 254 Further, tumor-specific markers, that are crucial for the understanding of the tumor biology, were 
255 shown to be specifically detected by USMI, enabling early cancer detection, therapy prediction,

256 and therapy monitoring. The first clinical studies confirmed feasibility of USMI for the detection 257 of VEGFR2 protein in human breast, ovarian, and prostate cancer. Continued efforts to improve 258 targeted contrast agents and specific imaging strategies for 2D and 3D imaging are expected to 259 further promote clinical translation of this powerful imaging technique and improve patient 260 management.

\section{Funding}

263 This work was supported by the LabEx DEVweCAN (ANR-10-LABX-0061) of the University of 264 Lyon, within the program "Investissements d'Avenir" (ANR-11-IDEX-0007) operated by the 265 French National Research Agency (ANR) and the French Ligue nationale contre le cancer. The 266 first author, Jennifer Wischhusen, was supported by the LabEx DEVweCAN (ANR-10-LABX267 0061) of the University of Lyon, within the program "Investissements d'Avenir" (ANR-11-IDEX268 0007) operated by the French National Research Agency (ANR), by the German-American 269 Fulbright Commission, by the France-Stanford Center for Interdisciplinary Studies, and by NIH R01CA155289. 


\section{References}

273 [1] Szabo TL. Diagnostic Ultrasound Imaging: Inside Out. 2nd ed. Academic Press; 2013.

274 [2] Duck FA, Baker AC, Starritt HC. Ultrasound in Medicine. Medical Science Series; 1998.

275 [3] Jain RK. Molecular regulation of vessel maturation. Nat Med 2003;9:685-93. 276 doi:10.1038/nm0603-685.

277 [4] Paefgen V, Doleschel D, Kiessling F. Evolution of contrast agents for ultrasound imaging 278 and ultrasound-mediated drug delivery. Front Pharmacol 2015;6. doi:10.3389/fphar.2015.00197.

279 [5] Feinstein SB, Cheirif J, Ten Cate FJ, Silverman PR, Heidenreich PA, Dick C, et al. Safety 280 and efficacy of a new transpulmonary ultrasound contrast agent: initial multicenter clinical 281 results. J Am Coll Cardiol 1990;16:316-24.

282 [6] Unnikrishnan S, Klibanov AL. Microbubbles as Ultrasound Contrast Agents for 283 Molecular Imaging: Preparation and Application. American Journal of Roentgenology 284 2012;199:292-9. doi:10.2214/AJR.12.8826.

285 [7] Caskey CF, Hu X, Ferrara KW. Leveraging the power of ultrasound for therapeutic 286 design and optimization. J Control Release 2011;156:297-306. 287 doi:10.1016/j.jconrel.2011.07.032.

288 [8] Klibanov AL, Hughes MS, Marsh JN, Hall CS, Miller JG, Wible JH, et al. Targeting of 289 ultrasound contrast material. An in vitro feasibility study. Acta Radiol Suppl 1997;412:113-20.

290 [9] Tranquart F, Arditi M, Bettinger T, Frinking P, Hyvelin JM, Nunn A, et al. Ultrasound 291 Contrast Agents For Ultrasound Molecular Imaging. Z Gastroenterol 2014;52:1268-76. 292 doi:10.1055/s-0034-1384999.

293 [10] Pochon S, Tardy I, Bussat P, Bettinger T, Brochot J, von Wronski M, et al. BR55: a 294 lipopeptide-based VEGFR2-targeted ultrasound contrast agent for molecular imaging of 295 angiogenesis. Invest Radiol 2010;45:89-95. doi:10.1097/RLI.0b013e3181c5927c.

296 [11] Deshpande N, Needles A, Willmann JK. Molecular ultrasound imaging: current status and future directions. Clin Radiol 2010;65:567-81. doi:10.1016/j.crad.2010.02.013.

299 2011;1:503-19. doi:10.1098/rsfs.2011.0019.

300 [13] Kiessling F. Science to Practice: Multiparametric Molecular and Functional US Imaging 301 Goes Three-dimensional. Radiology 2017;282:307-9. doi:10.1148/radiol.2016161455.

302 [14] Abou-Elkacem L, Bachawal SV, Willmann JK. Ultrasound molecular imaging: Moving 303 toward clinical translation. Eur J Radiol 2015;84:1685-93. doi:10.1016/j.ejrad.2015.03.016.

306 [16] Koczera P, Appold L, Shi Y, Liu M, Dasgupta A, Pathak V, et al. PBCA-based polymeric 307 microbubbles for molecular imaging and drug delivery. J Control Release 2017;259:128-35. 308 doi:10.1016/j.jconrel.2017.03.006.

309 [17] Güvener N, Appold L, de Lorenzi F, Golombek SK, Rizzo LY, Lammers T, et al. Recent 310 advances in ultrasound-based diagnosis and therapy with micro- and nanometer-sized 311 formulations. Methods 2017. doi:10.1016/j.ymeth.2017.05.018. 
[18] Alkan-Onyuksel H, Demos SM, Lanza GM, Vonesh MJ, Klegerman ME, Kane BJ, et al. Development of inherently echogenic liposomes as an ultrasonic contrast agent. J Pharm Sci 1996;85:486-90. doi:10.1021/js950407f.

[19] Perera RH, Hernandez C, Zhou H, Kota P, Burke A, Exner AA. Ultrasound Imaging Beyond the Vasculature with New Generation Contrast Agents. Wiley Interdiscip Rev Nanomed Nanobiotechnol 2015;7:593-608. doi:10.1002/wnan.1326.

[20] Paul S, Nahire R, Mallik S, Sarkar K. Encapsulated microbubbles and echogenic liposomes for contrast ultrasound imaging and targeted drug delivery. Comput Mech 2014;53:413-35. doi:10.1007/s00466-013-0962-4.

[21] Wilson KE, Wang TY, Willmann JK. Acoustic and Photoacoustic Molecular Imaging of Cancer. J Nucl Med 2013;54:1851-4. doi:10.2967/jnumed.112.115568.

[22] Wischhusen J, Padilla F. Microbubble Enzyme-Linked Immunosorbent Assay for the Detection of Targeted Microbubbles in in Vitro Static Binding Assays. Ultrasound Med Biol 2017;43:1506-19. doi:10.1016/j.ultrasmedbio.2017.03.004.

[23] Holmes K, Roberts OL, Thomas AM, Cross MJ. Vascular endothelial growth factor receptor-2: structure, function, intracellular signalling and therapeutic inhibition. Cell Signal 2007;19:2003-12. doi:10.1016/j.cellsig.2007.05.013.

[24] Willmann JK, Paulmurugan R, Chen K, Gheysens O, Rodriguez-Porcel M, Lutz AM, et al. US imaging of tumor angiogenesis with microbubbles targeted to vascular endothelial growth factor receptor type 2 in mice. Radiology 2008;246:508-18. doi:10.1148/radiol.2462070536.

[25] Pysz MA, Foygel K, Rosenberg J, Gambhir SS, Schneider M, Willmann JK. Antiangiogenic cancer therapy: monitoring with molecular US and a clinically translatable contrast agent (BR55). Radiology 2010;256:519-27. doi:10.1148/radiol.10091858.

[26] Zhang H, Tam S, Ingham ES, Mahakian LM, Lai C-Y, Tumbale SK, et al. Ultrasound molecular imaging of tumor angiogenesis with a neuropilin-1-targeted microbubble. Biomaterials 2015;56:104-13. doi:10.1016/j.biomaterials.2015.03.043.

[27] Leguerney I, Scoazec J-Y, Gadot N, Robin N, Pénault-Llorca F, Victorin S, et al. Molecular ultrasound imaging using contrast agents targeting endoglin, vascular endothelial growth factor receptor 2 and integrin. Ultrasound Med Biol 2015;41:197-207. doi:10.1016/j.ultrasmedbio.2014.06.014.

[28] Zhang H, Ingham ES, Gagnon MKJ, Mahakian LM, Liu J, Foiret JL, et al. In vitro characterization and in vivo ultrasound molecular imaging of nucleolin-targeted microbubbles. Biomaterials 2017;118:63-73. doi:10.1016/j.biomaterials.2016.11.026.

[29] Hua S. Targeting sites of inflammation: intercellular adhesion molecule-1 as a target for novel inflammatory therapies. Front Pharmacol 2013;4:127. doi:10.3389/fphar.2013.00127.

[30] El Kaffas A, Smith K, Pradhan P, Machtaler S, Wang H, von Eyben R, et al. Molecular Contrast-Enhanced Ultrasound Imaging of Radiation-Induced P-Selectin Expression in Healthy Mice Colon. Int J Radiat Oncol Biol Phys 2017;97:581-5. doi:10.1016/j.ijrobp.2016.10.037.

[31] Yeh JS-M, Sennoga CA, McConnell E, Eckersley R, Tang M-X, Nourshargh S, et al. Quantitative ultrasound molecular imaging. Ultrasound Med Biol 2015;41:2478-96. doi:10.1016/j.ultrasmedbio.2015.04.011. 
[32] Curaj A, Wu Z, Fokong S, Liehn EA, Weber C, Burlacu A, et al. Noninvasive molecular ultrasound monitoring of vessel healing after intravascular surgical procedures in a preclinical setup. Arterioscler Thromb Vasc Biol 2015;35:1366-73. doi:10.1161/ATVBAHA.114.304857. [33] Zhang Y-J, Bai D-N, Du J-X, Jin L, Ma J, Yang J-L, et al. Ultrasound-guided imaging of junctional adhesion molecule-A-targeted microbubbles identifies vulnerable plaque in rabbits. Biomaterials 2016;94:20-30. doi:10.1016/j.biomaterials.2016.03.049.

[34] Daeichin V, Kooiman K, Skachkov I, Bosch JG, Theelen TL, Steiger K, et al. Quantification of Endothelial av $\beta 3$ Expression with High-Frequency Ultrasound and Targeted Microbubbles: In Vitro and In Vivo Studies. Ultrasound Med Biol 2016;42:2283-93. doi:10.1016/j.ultrasmedbio.2016.05.005.

[35] Couture O, Dransart E, Dehay S, Nemati F, Decaudin D, Johannes L, et al. Tumor delivery of ultrasound contrast agents using Shiga toxin B subunit. Mol Imaging 2011;10:13543.

[36] Guo S, Shen S, Wang J, Wang H, Li M, Liu Y, et al. Detection of high-risk atherosclerotic plaques with ultrasound molecular imaging of glycoprotein IIb/IIIa receptor on activated platelets. Theranostics 2015;5:418-30. doi:10.7150/thno.10020.

[37] Nakatsuka MA, Barback CV, Fitch KR, Farwell AR, Esener SC, Mattrey RF, et al. In vivo ultrasound visualization of non-occlusive blood clots with thrombin-sensitive contrast agents. Biomaterials 2013;34:9559-65. doi:10.1016/j.biomaterials.2013.08.040.

[38] Shim CY, Liu YN, Atkinson T, Xie A, Foster T, Davidson BP, et al. Molecular Imaging of Platelet-Endothelial Interactions and Endothelial von Willebrand Factor in Early and MidStage Atherosclerosis. Circ Cardiovasc Imaging 2015;8:e002765. doi:10.1161/CIRCIMAGING.114.002765.

[39] Bachawal SV, Jensen KC, Wilson KE, Tian L, Lutz AM, Willmann JK. Breast Cancer Detection by B7-H3-Targeted Ultrasound Molecular Imaging. Cancer Res 2015;75:2501-9. doi:10.1158/0008-5472.CAN-14-3361.

[40] Barua A, Yellapa A, Bahr JM, Adur MK, Utterback CW, Bitterman P, et al. Interleukin 16- (IL-16-) Targeted Ultrasound Imaging Agent Improves Detection of Ovarian Tumors in Laying Hens, a Preclinical Model of Spontaneous Ovarian Cancer. Biomed Res Int 2015;2015. doi:10.1155/2015/567459.

[41] Koonce NA, Levy J, Hardee ME, Jamshidi-Parsian A, Vang KB, Sharma S, et al. Targeting Artificial Tumor Stromal Targets for Molecular Imaging of Tumor Vascular Hypoxia. PLoS ONE 2015;10:e0135607. doi:10.1371/journal.pone.0135607.

[42] Tsuruta JK, Schaub NP, Rojas JD, Streeter J, Klauber-DeMore N, Dayton P. Optimizing ultrasound molecular imaging of secreted frizzled related protein 2 expression in angiosarcoma. PLoS ONE 2017;12:e174281. doi:10.1371/journal.pone.0174281.

[43] Zlitni A, Yin M, Janzen N, Chatterjee S, Lisok A, Gabrielson KL, et al. Development of prostate specific membrane antigen targeted ultrasound microbubbles using bioorthogonal chemistry. PLoS ONE 2017;12:e0176958. doi:10.1371/journal.pone.0176958. 
[44] Wischhusen J, Wilson K, Delcros J, Molina-Peña R, Gibert B, Jiang S, et al. Ultrasound molecular imaging as a non-invasive companion diagnostic for netrin-1 interference therapy in breast cancer. Theranostics 2018; 8(18):5126-5142. doi:10.7150/thno.27221.

[45] Ehling J, Misiewicz M, von Stillfried S, Möckel D, Bzyl J, Pochon S, et al. In situ validation of VEGFR-2 and $\alpha \vee \beta 3$ integrin as targets for breast lesion characterization. Angiogenesis 2016;19:245-54. doi:10.1007/s10456-016-9499-4.

[46] Wang H, Felt SA, Machtaler S, Guracar I, Luong R, Bettinger T, et al. Quantitative Assessment of Inflammation in a Porcine Acute Terminal Ileitis Model: US with a Molecularly Targeted Contrast Agent. Radiology 2015:142478. doi:10.1148/radiol.2015142478.

[47] Ferrante EA, Pickard JE, Rychak J, Klibanov A, Ley K. Dual targeting improves microbubble contrast agent adhesion to VCAM-1 and P-selectin under flow. J Control Release 2009;140:100-7. doi:10.1016/j.jconrel.2009.08.001.

[48] Warram JM, Sorace AG, Saini R, Umphrey HR, Zinn KR, Hoyt K. A triple-targeted ultrasound contrast agent provides improved localization to tumor vasculature. J Ultrasound Med 2011;30:921-31.

[49] Smeenge M, Tranquart F, Mannaerts CK, de Reijke TM, van de Vijver MJ, Laguna MP, et al. First-in-Human Ultrasound Molecular Imaging With a VEGFR2-Specific Ultrasound Molecular Contrast Agent (BR55) in Prostate Cancer: A Safety and Feasibility Pilot Study. Invest Radiol 2017;52:419-27. doi:10.1097/RLI.0000000000000362.

[50] Willmann JK, Bonomo L, Carla Testa A, Rinaldi P, Rindi G, Valluru KS, et al. Ultrasound Molecular Imaging With BR55 in Patients With Breast and Ovarian Lesions: First-inHuman Results. J Clin Oncol 2017;35:2133-40. doi:10.1200/JCO.2016.70.8594. 
Figure 1: Microbubble (MB) functionalized with an antibody ligand for molecular targeting.

Figure 2: Ultrasound molecular imaging (USMI) with microbubbles (MBs) targeting a biomarker of the tumor endothelium.

Figure 3: Alternative ultrasound contrast agents for intravascular and extravascular molecular imaging. (A) Perfluorocarbon emulsion nanodroplets which can be vaporized, (B) echogenic liposomes, where air pockets are present in the phospholipid bilayer, $(\mathrm{C})$ nanobubbles which fuse into echogenic microbubbles, (D) solid nanoparticles with gas-filled pores.

Figure 4: Ultrasound molecular imaging (USMI) of netrin-1 in human breast tumors using targeted microbubbles (MBs). Netrin-1-targeted MBs $\left(\mathrm{MBs}_{\mathrm{Netrin}-1}\right)$ or isotype control MBs

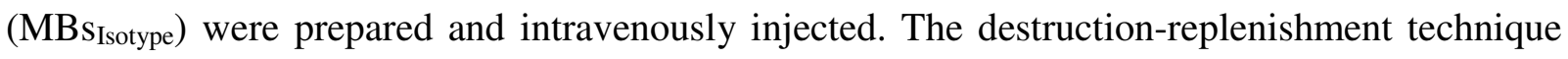
was applied (pre-burst acquisition 4 min after injection; burst 4.5 min after injection immediately followed by post-burst acquisition) and differential targeted enhancement in linear arbitrary units

434 (l.a.u.) is depicted in color over contrast-enhanced ultrasound (CEUS) images in the right 435 column. As a control, grey-scale B-mode images are depicted in the left column. To validate 436 novel netrin-1-targeted MBs, human netrin-1-expressing SKBR7 or negative MDA-MB-231 437 breast tumors were imaged. Targeted MBs specifically accumulated in netrin-1-positive tumors 438 only. 

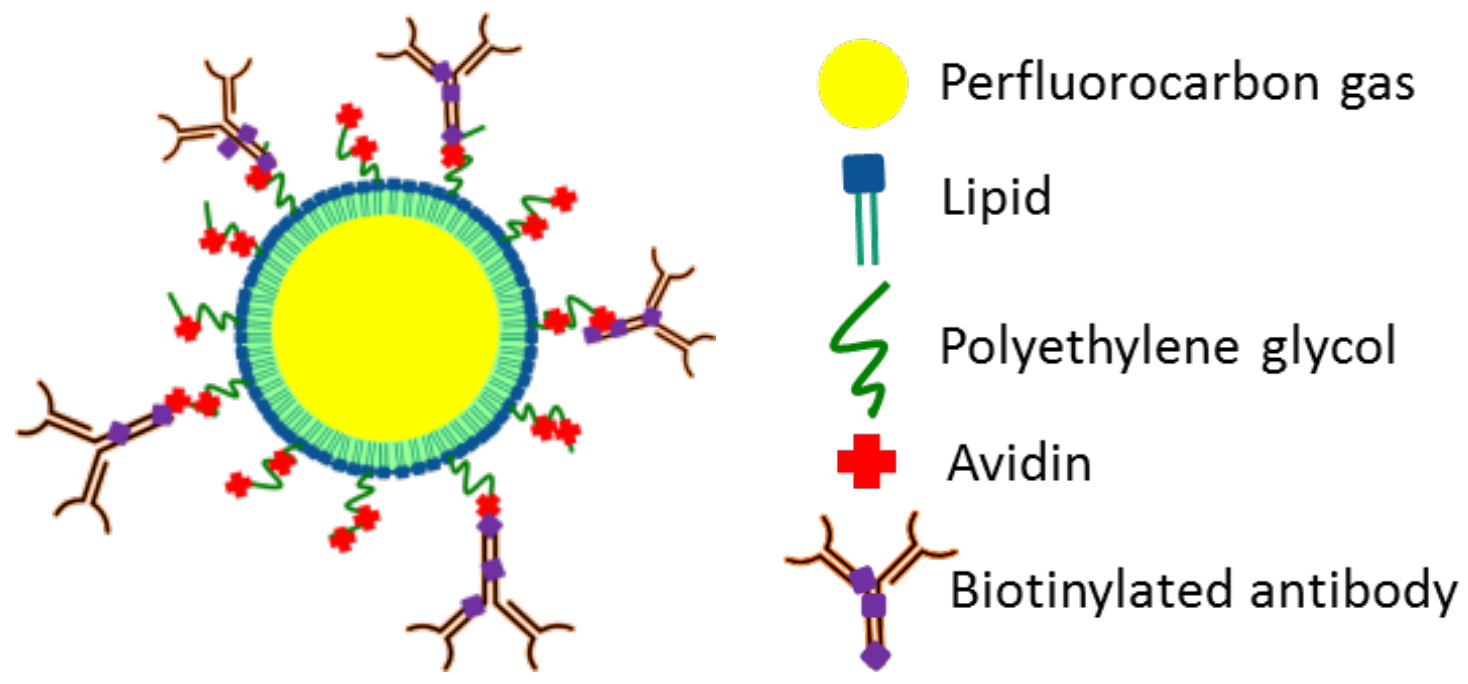

Figure 1: Microbubble (MB) functionalized with an antibody ligand for molecular targeting. 


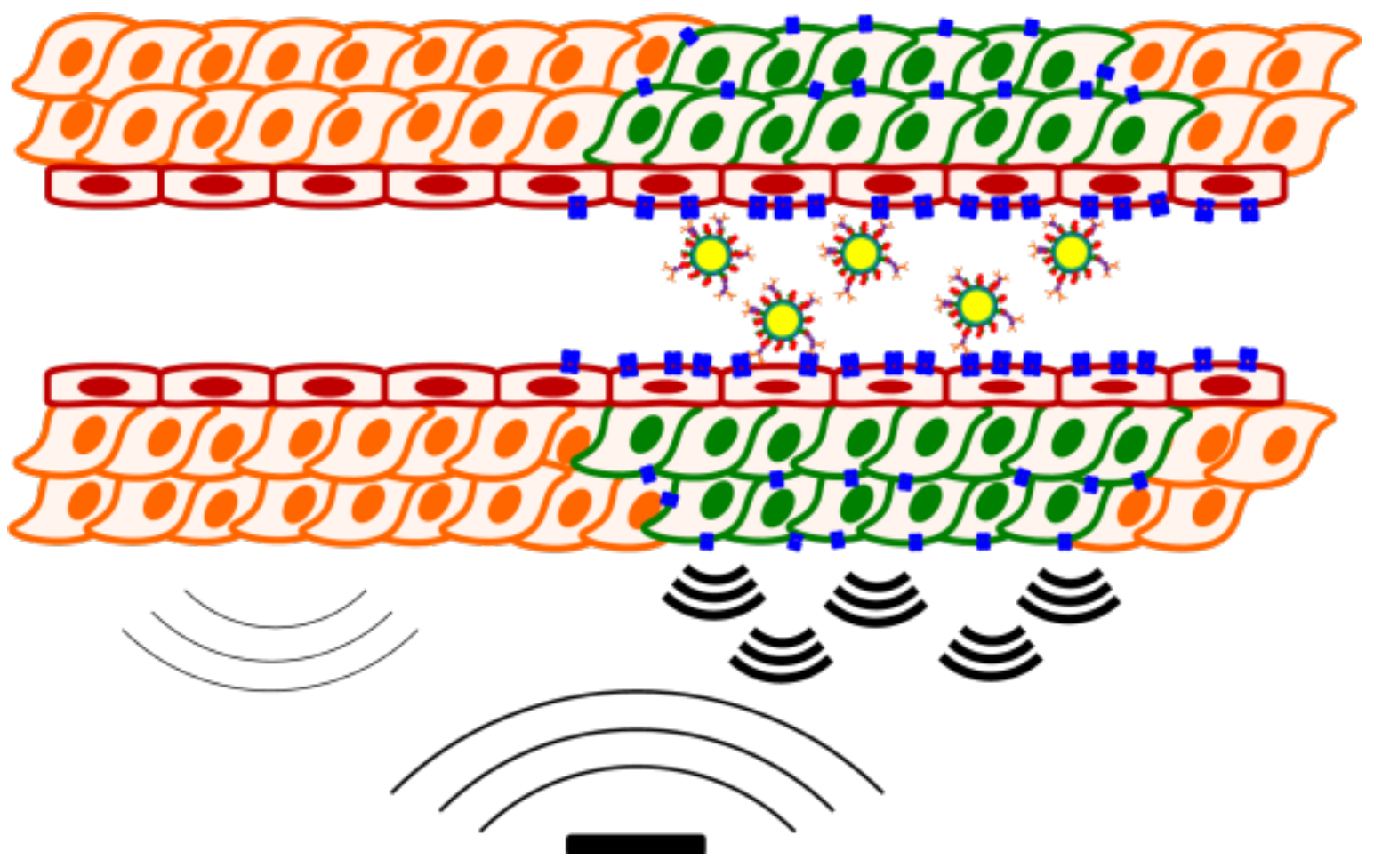

Normal cell

D. Tumor cell

$\varpi$ Endothelial cell

I. Vascular endothelial growth factor receptor 2 (VEGFR2)

Microbubble

$\approx$ Backscatter

こ Ultrasound device

Figure 2: Ultrasound molecular imaging (USMI) with microbubbles (MBs) targeting a biomarker of the tumor endothelium. 
B

Liquid perfluorocarbon

core

Phospholipid monolayer

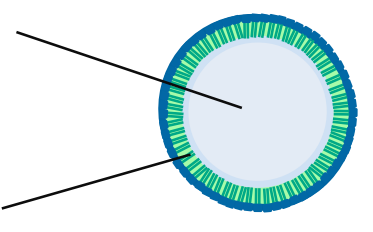

Nanodroplet 200 - $400 \mathrm{~nm}$

C

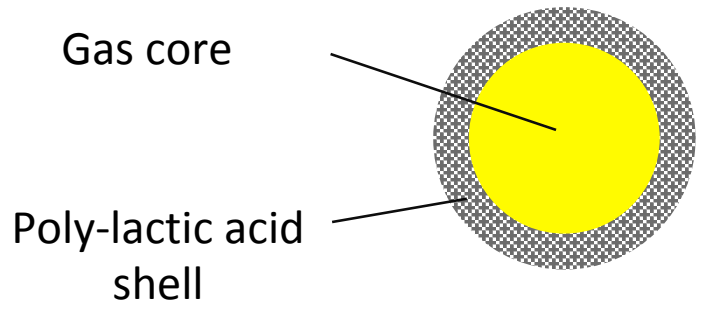

Nanobubble 40 - $200 \mathrm{~nm}$

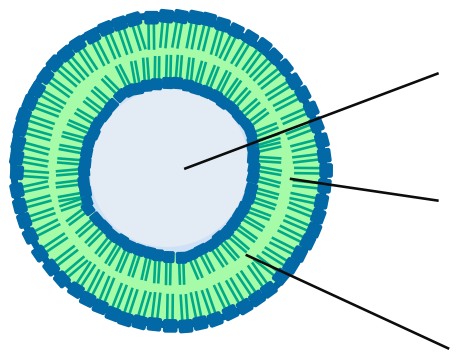

Liposome $20 \mathrm{~nm}-10 \mu \mathrm{m}$

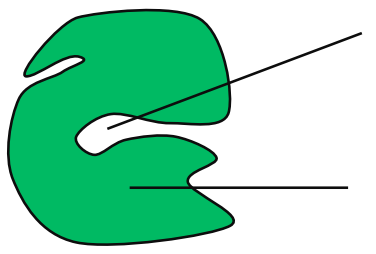

Gas pocket

Solid particle
Phospholipid bilayer

Air pockets
Aqueous core

D

\section{Solid nanoparticle 20 - 100 nm}

Figure 3: Alternative ultrasound contrast agents for intravascular and extravascular molecular imaging. (A) Perfluorocarbon emulsion nanodroplets which can be vaporized, (B) echogenic liposomes, where air pockets are present in the phospholipid bilayer, (C) nanobubbles which fuse into echogenic microbubbles, (D) solid nanoparticles with gas-filled pores. 


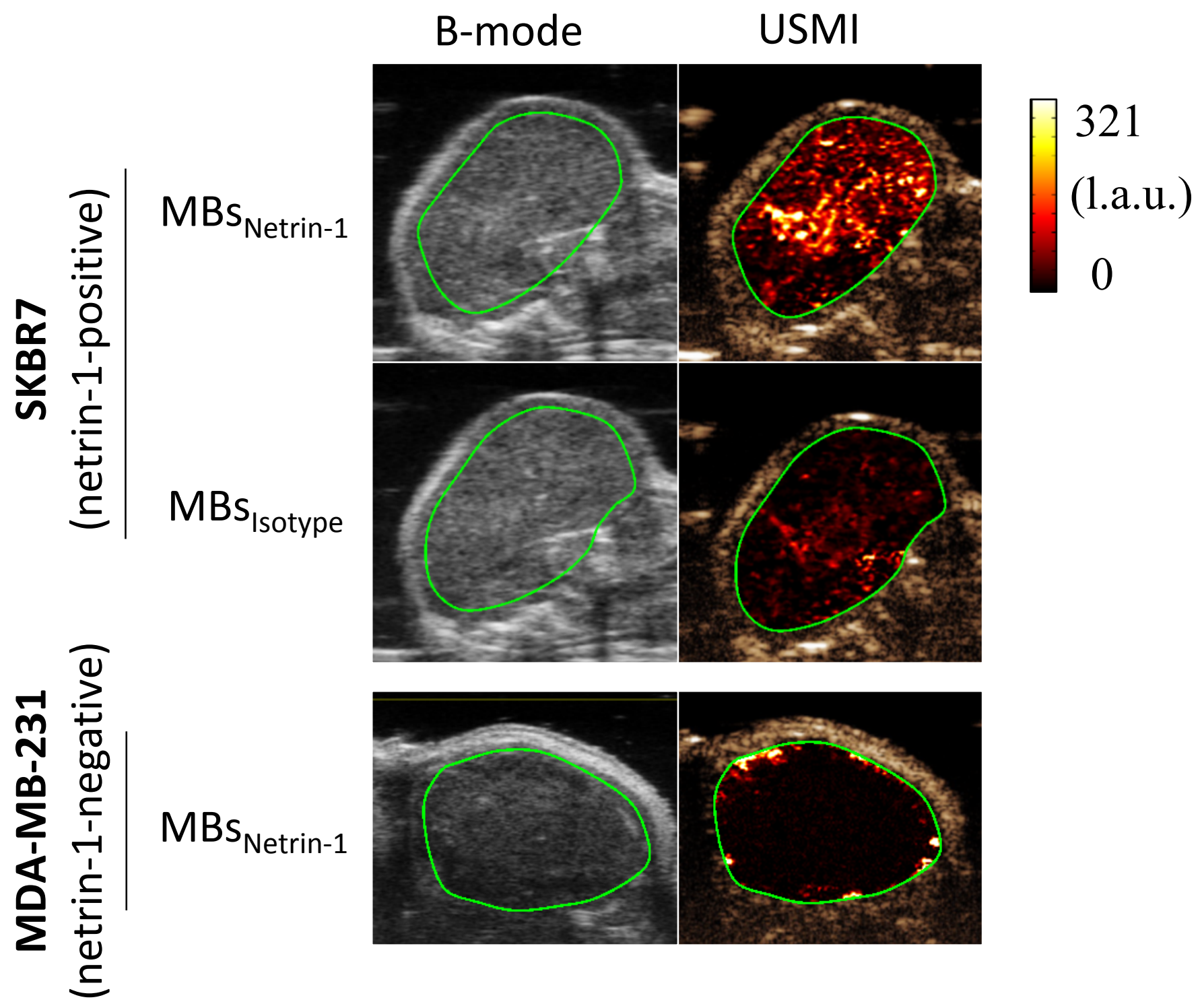

Figure 4: Ultrasound molecular imaging (USMI) of netrin-1 in human breast tumors using targeted microbubbles (MBs). Netrin-1-targeted $\mathrm{MBs}\left(\mathrm{MBs}_{\mathrm{Netrin}-1}\right)$ or isotype control MBs $\left(\mathrm{MBs}_{\text {Isotype }}\right)$ were prepared and intravenously injected. The destruction-replenishment technique was applied (pre-burst acquisition $4 \mathrm{~min}$ after injection; burst $4.5 \mathrm{~min}$ after injection immediately followed by post-burst acquisition) and differential targeted enhancement in linear arbitrary units (1.a.u.) is depicted in color over contrast-enhanced ultrasound (CEUS) images in the right column. As a control, grey-scale B-mode images are depicted in the left column. To validate novel netrin-1targeted MBs, human netrin-1-expressing SKBR7 or negative MDA-MB-231 breast tumors were imaged. Targeted MBs specifically accumulated in netrin-1-positive tumors only. 
